\title{
A Telephone-Based Dementia Care Management Intervention-Finding the Time to Listen
}

Jennifer Tjia, MD, MSCE

In this issue of JAMA Internal Medicine, Possin and colleagues ${ }^{1}$ report the results of their trial testing a telephone- and internetbased supportive care management program, the Care Ecosystem, for patients with dementia and their caregivers. In this

\section{$+$}

Author Audio Interview

$+$

Related articles model, care is delivered by unlicensed Care Team Navigators (CTNs) who are trained and supervised by a dementia specialist nurse, social worker, and pharmacist. Re-

sults of the trial indicate that the program improved patient quality of life, decreased caregiver depression and burden, and reduced emergency department visits. These results are especially impressive given the robust control intervention that provided access to existing, high-quality resources from the Family Caregiver Alliance, Alzheimer Association, and Area Agencies on Aging. These trial results offer hope to the estimated 16 million caregivers of patients living with dementia in the United States, ${ }^{2}$ many of whom have inadequate dementia care support. ${ }^{3}$

Dementia is well known to be a family disease. ${ }^{4}$ Caregivers are stressed, frustrated, and overburdened. ${ }^{3}$ Although the Care Ecosystem program is neither the first nor the only dementia care management program to demonstrate effectiveness, ${ }^{5}$ it is innovative and important because it demonstrates success in scaling services across geographic and health system boundaries, reaching urban and rural populations regardless of patients' insurer or clinician affiliation. Using a telephonic and webbased platform and demonstrating the ability to overcome language barriers (eg, Spanish and Cantonese speakers), the Care Ecosystem provides a major step forward not only for patients and their caregivers, but also for the health systems that are financially responsible for them during the continuing shift toward value-based payment structures.

The potential influence of this advance in clinical and care management struck close to home as I reflected on how such a program could have helped my own family navigate my father's decline due to dementia. Living 3000 miles away, my father was cared for by my elderly mother at home. Like many families living with dementia, we went through a journey together as my father progressed from the early stages of executive function loss to the late stages of complete dependence. I mostly experienced this odyssey remotely through the stories my mom shared on the telephone. Early on, these stories chronicled the symptoms of forgetfulness-"Can you believe he didn't water the plants again?!”-and apathy- "Why doesn't he want to exercise when I tell him to?” Through these conversations I realized how a skilled CTN could have allowed her to express frustration and fear while helping her to remain resilient. Eventually, as the calls became more serious, I real- ized that despite my medical knowledge, I was having difficulty helping my mother appreciate the risks of managing my father at home. In the setting of impulsiveness after he was hospitalized for a fall-related subdural hematoma, I saw the need for focused guidance from dementia-savvy clinicians who might help her implement realistic strategies. It is this type of situation in which an intervention of telephonic support could potentially prevent repeat hospitalization. During his final hospitalization for aspiration pneumonia, the calls with my mom shifted to arguments about whether he should stay in the hospital or return home. "He's getting so much attention from those nice doctors!" It was only in retrospect that I realized she was in desperate need of respite, but she did not have the words to state this need. A trained clinician would have likely spotted this and strategized appropriately. Lastly, near the end of his life, the calls were about his progressive weight loss and declining appetite. "He won’t eat," my mom would say. "I have to force him to eat more.” I didn't know how to help her understand that he was dying, and I would have been grateful for the assistance of a health care team member who had been with her throughout the journey.

As a board-certified geriatric and palliative care specialist, I watched and listened to this story unfold with the knowing perspective of a trained professional, intellectually observing him march through the well-worn stages of dementia. However, as a daughter, with my own reactions to my mom's difficulty in accepting his diagnosis, I struggled to provide the empathetic listening and communication that she so desperately needed. She needed a team of dementia-care specialists equipped to listen to her concerns, communicate support and empathy, educate her about the disease course, and provide expert medical advice for her, my dad, and their non-dementia specialist clinical care team.

I believe there are several reasons why the Care Ecosystem design was effective. First, listening is healing. In the Care Ecosystem intervention, CTNs were hired for strong communication and listening skills, and they were trained to first respond to caregiver immediate needs and then to screen for common problems and provide personalized support. Caregivers need someone who can empathically listen to their dilemmas, frustrations, and stories about their loved one, who is slipping away. With a strong foundation of communication, caregivers are likely more able to absorb the benefits of education and advice on how to navigate the critical transitions in the disease course.

Second, the telephone can be an effective lifeline. Caregivers are often constrained in their ability to travel given the responsibilities of maintaining the safety of their person with dementia, which causes isolation and increases caregiver stress. Even 1 call per month, the baseline frequency of calls by Care 
Ecosystem CTNs, seemed to make a difference to caregivers in the intervention. Although other research shows that caregivers may be reluctant to share the difficult aspects of caring with outsiders and prefer to obtain information from their own health care professionals, ${ }^{6}$ it seems that the Care Ecosystem model overcame these barriers, with more than $90 \%$ of caregivers accepting the complete Care Ecosystem program.

Finally, in a world where geographic, economic, and health system boundaries can dictate access to care, the Care Ecosystem was offered without regard to these traditional borders. Patients and caregivers could receive Care Ecosystem support regardless of whether they received services in a closed, capitated system, from a single CTN paid via the Medicare fee-for-service program, or from another care delivery configuration. The new Medicare billing code to provide reimbursement for monthly chronic care management by clinical staff $^{7}$ may help health systems create and offer similar pro- grams. However, given the workforce constraints limiting the availability of dementia care specialists nationwide, health care and policy leaders need to strongly consider ways to leverage existing dementia support programs through innovative payment mechanisms or carve-outs to contract with existing services.

Dementia is a public health challenge, a family disease, and a major strain on our health care system. Possin and colleagues ${ }^{1}$ have provided evidence that achieving the goals of the triple aim of improving care, health outcomes, and reducing cost can be met by providing dementia training to clinically supervised, unlicensed CTNs in a scalable way. Policy makers and health system leaders can take notice and support access to this model so that the numerous families who are living with dementia can have the possibility of better care and outcomes that result from expert support, knowledge, and tailored care.
ARTICLE INFORMATION

Author Affiliation: University of Massachusetts Medical School, Worcester, Massachusetts.

Corresponding Author: Jennifer Tjia, MD, MSCE, University of Massachusetts Medical School, 368 Plantation St, 6th Floor, Worcester, MA 01605 (jennifer.tjia@umassmed.edu).

Published Online: September 30, 2019. doi:10.1001/jamainternmed.2019.4111

Conflict of Interest Disclosures: Dr Tjia reports grants from the National Institutes of Health and the Cambia Health Foundation, personal fees from the Donaghue Foundation, and personal fees and nonfinancial support from CVS Health outside the submitted work.

Additional Contributions: The author thanks all those described herein for granting permission to publish this information and Jane L. Givens, MD, for reviewing an early draft of this article.

\section{REFERENCES}

1. Possin KL, Merrilees JJ, Dulaney S, et al. Effect of collaborative dementia care via telephone and internet on quality of life, caregiver well-being, and health care use: the care ecosystem randomized clinical trial [published online September 30, 2019]. JAMA Intern Med. doi:10.1001/jamainternmed.2019. 4101

2. Alzheimer's Association. Alzheimer's disease caregivers fact sheet. http://act.alz.org/site/ DocServer/caregivers_fact_sheet.pdf?doclD=3022. Updated March 2019. Accessed on July 19, 2019.

3. Brodaty H, Donkin M. Family caregivers of people with dementia. Dialogues Clin Neurosci. 2009;11(2):217-228.

4. Epstein-Lubow G. A family disease: witnessing firsthand the toll that dementia takes on caregivers. Health Aff (Millwood). 2014;33(4):708-711. doi:10. 1377/hlthaff.2012.1351
5. Goeman D, Renehan E, Koch S. What is the effectiveness of the support worker role for people with dementia and their carers? A systematic review. BMC Health Serv Res. 2016;16:285. doi:10. 1186/s12913-016-1531-2

6. Slaboda J, Fail R, Noman G, Meier D. A study of family caregiver burden and the imperative of practice change to address family caregivers' unmet needs. Health Affairs blog. https://www. healthaffairs.org/do/10.1377/hblog20180105. 914873/full/. Accessed July 19, 2019.

7. Centers for Medicare and Medicaid. Medicare learning network: chronic care management services. https://www.cms.gov/Outreach-andEducation/Medicare-Learning-Network-MLN/ MLNProducts/Downloads/

ChronicCareManagement.pdf. Accessed on July 19. 2019. 